\title{
Could Early Identification of Changes in Olfactory Function Be an Indicator of Preclinical Neurodegenerative Disease? A Systematic Review
}

Rikki L. Winchester (D) - Kathy Martyn (D)

Received: May 2, 2020 / Published online: June 11, 2020

(c) The Author(s) 2020
Digital features To view digital features for this article go to https://doi.org/10.6084/m9.figshare.12377144.

Electronic supplementary material The online version of this article (https://doi.org/10.1007/s40120020-00199-z) contains supplementary material, which is available to authorized users.

R. L. Winchester $(\bowtie)$

Brighton and Sussex Medical School, University of

Sussex, Brighton, England

e-mail: rikkiwinchester23@gmail.com

K. Martyn

University of Brighton, Brighton, England
Methods: A systematic review was performed via Medline on 17 October 2019 using the search terms and Boolean operators 'Dementia OR Alzheimer's AND olfaction AND cognitive impairment' yielding 111 results. These were then screened using inclusion/exclusion criteria alongside a PICO strategy. After titles, abstracts and full text were screened, nine articles were included in the review and critically appraised using the AXIS and CASP tools.

Results: Significant correlations are demonstrated between olfactory impairment (OI) and cognitive decline. However, there were limitations of many of the studies in that confounders such as head trauma, upper respiratory infection (URTI) and smoking history were not considered. The majority of the studies also used an olfactory screening tool that was not designed for the population being examined.

Conclusion: Despite improvements in olfactory testing needing to be implemented, OI is clearly impaired in neurodegenerative disease across a multitude of ages and cultures, offering an early marker of future cognitive decline. As a result of the heterogenous nature of the included studies, there is a further need for future research to ensure the sensitivity, validity and reliability of implementing olfactory testing as an early marker of future cognitive decline.

Keywords: Alzheimer's disease; Cognitive impairment; Dementia; Diagnosis; Olfactory impairment; Preclinical; Screening 


\section{Key Summary Points}

\section{Background}

Alzheimer's accounts for $60-80 \%$ of all dementias, affecting 850,000 in the UK and predicted to increase to 1.6 million by 2040.

Diagnosis only after significant neuronal pathology has occurred, treatment focused on symptom control only.

Olfactory nerve pathology may occur before cognitive symptoms manifest facilitating preclinical diagnosis and opportunity to develop novel therapeutics to inhibit disease progression.

\section{Aims}

To critically appraise relevant literature to establish whether olfactory testing provides a suitably accurate preclinical biomarker of Alzheimer's disease for clinical use, and to make recommendations for future research.

\section{Key findings}

Olfactory impairment (OI) is implicated in cognitive decline. However, despite homogeneity of conclusions, limitations of study design result in conclusions that may not completely isolate neurodegenerative pathology as driver of OI. It is therefore recommended that future studies use a minimum set of inclusion/exclusion criteria for increasingly accurate associations.

\section{INTRODUCTION}

Alzheimer's disease (AD) is a debilitating neurodegenerative condition that accounts for $60-80 \%$ of all cases of dementia [1]. It currently affects 850,000 people in the UK, with estimates rising to 1.6 million by 2040 [2]. This inflation is partly attributable to the increase in population size and the continual rise in average life expectancy [3]. Compounding this, despite an evolving understanding of disease pathogenesis, current treatment for $\mathrm{AD}$ is mainly focused on symptom control and does little to nothing in halting disease progression. Fundamentally, a growing number of the population are reaching an age in which the risk of developing a neurodegenerative disease becomes significantly more likely, with no way to prevent it.

Arguably the greatest challenge in AD management is diagnosis. In 1983, the National Institute of Neurological and Communicative Disorders and Stroke (NINCDS) and the Alzheimer's Disease and Related Disorders Association (ADRDA) convened a meeting with the purpose of establishing criteria to aid in the clinical diagnosis of $\mathrm{AD}$ [4]. This report addressed factors such as clinical history, examination, neuropsychological testing and laboratory assessments that have proven to be relatively successful over time with a sensitivity of $81 \%$ and specificity of $70 \%$ [5]. A weakness of the criteria is its reliance on clinical features that could only provide a 'probable' diagnosis of $\mathrm{AD}$, retrospectively proven via histopathological confirmation. Since then, there have been continued developments in the understanding of $\mathrm{AD}$, and in 2007 the National Institute on Aging and the Alzheimer's Association (NIA-AA) began work on updating these criteria in response to recent innovations. These included magnetic resonance imaging (MRI), positron emission tomography (PET) and cerebrospinal fluid (CSF) assays to consider biomarkers such as tau and amyloid- $\beta\left(\mathrm{A} \beta_{42}\right)$ [6]. With the introduction of these biomarkers, there has been a shift in the concept of AD. This led to the International Working Group (IWG) proposing a new lexicon for both the medical and research community to account for the broader diagnostic coverage of AD [7]. The 2011 research framework published by the NIA-AA has separate diagnostic guidelines; one for clinical diagnosis [8] and another for preclinical AD [9]. The latter is designed exclusively for researchers to share a common language when testing hypotheses. This research framework is 
important because $\mathrm{AD}$ can be defined by either post-mortem examination or in vivo biomarkers, fundamentally shifting the possibility of diagnosis away from the clinical manifestations experienced by the patient and towards a biological construct. Jack et al. state that the shift from a syndromal to a biological definition of $\mathrm{AD}$ is a logical step forward because it facilitates enhanced understanding of the mechanisms underlying disease progression and that diseasemodifying interventions must engage a target defined biologically [10]. This is an essential step in the goal of designing interventions that can inhibit pathological mechanisms before symptoms become clinically evident.

However, Jack et al. state the current limitations of biomarkers. There can be a discordance between CSF and imaging, an example being the concentration of $A \beta_{42}$ in CSF not correlating with that seen via PET scanning in a linear fashion. Rather, it is 'L-shaped' and therefore suggests a temporal offset between the two. Tau PET ligand binding seems to show a more linear association with CSF phosphorylated tau (Ptau); however, this seems to plateau later in the disease while the PET signal continues to rise. It is also important to consider that a large majority of current PET and CSF data is based on participants recruited from tertiary settings rather than the community. Evidence showing that a clinic-based population differs from a community-based one and therefore conclusions may not be transferrable to a wider population [11].

In the UK, the National Institute for Health and Care Excellence (NICE) recommends that diagnosis is made by considering the patient's history, symptoms and examination alongside blood and urine tests to exclude an organic cause of cognitive decline. It is further recommended that cognitive testing such as the 10-point cognitive screener is used, which can improve diagnostic accuracy [12]. However, AD cannot be ruled out on the basis of a normal cognitive score. A suspected rapidly progressive dementia warrants a referral to neurological services where potential biomarkers of $\mathrm{AD}$ can be examined via CSF. This is an invasive procedure and therefore uncomfortable for the patient. It was previously thought that to diagnose $\mathrm{AD}$ there must be evidence of impairment in both memory and one other cognitive domain that are independent of organic causes. However, there are several non-amnestic presentations of $\mathrm{AD}$ such as the syndrome of posterior cortical atrophy [8, 13] that mean language difficulties can be taken as the primary deficit alongside one other such as visuospatial problems. Following this, if the diagnosis is still uncertain and $\mathrm{AD}$ is suspected, NICE recommends the use of imaging such as fluorodeoxyglucose-positron emission tomographyCT (FDG-PET), or perfusion single-photon emission CT (SPECT) and MRI [14]. NICE further states that $\mathrm{AD}$ cannot be ruled out solely on the basis of imaging results. Fundamentally, despite many methods to make an $\mathrm{AD}$ diagnosis more likely, there is no definitive way to consistently achieve it, and by the time symptoms become clinically evident, significant neuronal pathology has occurred. The window of opportunity has closed, and progressive cognitive decline appears inevitable.

Despite this, neurofibrillary tangles of the olfactory bulb are an early pathological feature of $\mathrm{AD}$. A growing body of research has shown a clear correlation between early olfactory changes and mild cognitive impairment (MCI), which is a recognised checkpoint on the way to $\mathrm{AD}[15,16]$. This is not a novel concept. Changes in olfactory function in $\mathrm{AD}$ have been observed as early as 1974 [17], but have not made their way into diagnostic criteria because of a number of challenges. Confounding factors such as smoking, stroke and upper respiratory tract infection (URTI) are among factors that can contribute to olfactory impairment (OI), meaning that it is difficult to determine the extent to which OI is affected by neurodegenerative pathology. It is also essential to consider that olfactory function declines as a normal part of aging. Murphy et al. illustrate that on average, $24.5 \%$ of those aged $53-97$ had some degree of OI [18], with Doty et al. demonstrating that more than $75 \%$ of healthy individuals aged 80 years or older had OI [19].

Like all physiological mechanisms of the human body, the principal role of olfaction is survival. Evidence shows that anosmics have a three times higher risk of experiencing a 
hazardous event when compared to normosmics [20], with approximately $50 \%$ of anosmics stating they had accidentally eaten spoiled or rotten food [21]. Olfaction guides one's perception away from ingesting hazardous substances such as smoke, toxins and microbial threats and towards items that imply a survival benefit such as nutritious foods. Moreover, it is such an important aspect of survival that it is an independent variable in predicting 5-year mortality despite accounting for confounders such as nutrition and neurodegenerative disease $[22,23]$.

Olfaction also has a strong link with emotional memory and quality of life (QOL). Sullivan et al. describe how the link between the olfactory nerve and the limbic system bypasses the thalamus, and therefore the cortex, to provide direct access to a region of memory and emotional processing [24]. Odours encountered during development are linked to positive/ negative emotions, which are triggered when re-encountered, suggesting that the olfactory nerve is a unique gateway to a part of ourselves that remains forever inaccessible when dysfunctional. This inevitably has a dramatic impact on our sense of wellbeing, safety, pleasure, appetite and overall QOL. Miwa et al. demonstrate that participants with OI stated a $25 \%$ reduction in QOL with areas such as safety in recognising noxious odours such as gas leaks, personal hygiene and lack of food enjoyment among the primary concerns [25]. Despite this, it seems that OI is an understated and neglected modality. Murphy et al. show that only $9.5 \%$ of 2491 participants reported olfactory impairment despite a prevalence of $24.5 \%$ when tested [18]. This is supported by further work by Doty et al. in patients with $\mathrm{AD}$ in which only $6 \%$ complained of OI despite $90 \%$ having impairment when tested [26]. The Australian Medical Association guidelines state that anosmia represents only 5\% impairment to the individual [27]; however, when considering the reduced QOL, increased risk of harm and mortality, it could be an area in which increased awareness could lead to a happier and healthier life.

Despite the challenges of assessing OI, correlations have been demonstrated in a variety of neurodegenerative diseases such as $\operatorname{AD}[28,29]$,
Parkinson's disease (PD) [30-32] and dementia with Lewy bodies (DLB) [33, 34]. Prevalence of OI in these conditions is also extremely high, seen in up to $100 \%$ of patients with $\mathrm{AD}$ and $90 \%$ of those with PD $[35,36]$. Testing is relatively cheap, quick and non-invasive. If testing could consistently predict future cognitive decline across a wide variety of cultures, it would provide a valuable preclinical marker of disease. If simple tests were administered in the context of the NHS Health Check, it is possible that early interventions could be designed and implemented to either dramatically slow disease progression or halt it entirely. This makes for an enticing proposition, with Alzheimer's UK stating that if we could delay dementia onset by 5 years, 30,000 lives could be saved each year [2]. The fundamental question becomes: what steps are required to utilise the increased diagnostic power that olfactory testing has to offer?

The main aims of this review were to critically appraise relevant literature to establish whether olfactory testing provides an accurate preclinical biomarker of $\mathrm{AD}$; to critically appraise the methods in selected articles to understand if conclusions are accurate enough for use in clinical practice and to highlight limitations in current olfactory testing to guide further research.

\section{NECESSITY OF SYSTEMATIC REVIEWS AND EVIDENCE-BASED MEDICINE}

High-quality research is the foundation on which healthcare professionals harness the necessary knowledge on the safety and efficacy of clinical interventions to make optimal decisions for the benefit of their patients. However, with an ever-increasing body of scientific literature coupled with a reduction in clinician time, it becomes increasingly difficult to draw accurate conclusions. Systematic reviews were implemented in the late 1970s as a method to harness the almost exponential growth of clinical research during this time [37]. Fundamentally, amalgamating a wide body of empirical evidence on a specific research question into a 
succinct conclusion increases the effectiveness of evidence-based medicine.

Transparency is a foundational principle, allowing researchers to understand the methods used to examine the literature and make their own conclusions on data presented. The Preferred Reporting Items for Systematic Reviews and Meta-Analyses (PRISMA) statement was developed in 2009 and consists of a 27-item checklist and flow diagram that includes items deemed essential for transparent reporting. It provides a minimum set of reporting standards, facilitating a uniform quality in presented data. Explicit, systematic methodology, such as a priori inclusion/exclusion criteria, is designed to reduce bias and provide accurate conclusions on which optimal clinical decisions can be made.

\section{METHODOLOGY}

\section{Information Searches and Sources}

An initial Google Scholar search was performed, facilitating orientation into current research and relevant keywords that would subsequentially inspire final search criteria. There were limited articles on changes to both taste AND appetite in preclinical detection of $\mathrm{AD}$; however, there was a wide body of research on how OI correlates to $\mathrm{MCI}$ and $\mathrm{AD}$ and therefore formed the basis of subsequent search criteria.

As a result of this being an undergraduate systematic review constrained by time, Medline was selected as the primary database due to research showing that a significantly high proportion of relevant literature could be retrieved using this database $[38,39]$. Dunn et al. showed that Medline was one of the top two resources (alongside print and online journals) used by clinicians when searching for information on optimal patient care [40]. Further reasons include that all articles are peer-reviewed, only original research is published, articles must primarily target health professionals and researchers, and all content is freely available via PubMed.

\section{Olfactory Testing Criteria}

For the purpose of this review, only articles using the University of Pennsylvania Smell Identification Test (UPSIT) or Sniffin' Sticks test were included.

The UPSIT is a 40-item test comprising microencapsulated odorants which the participant releases via scratching and selecting the appropriate fragrance via four multiple-choice options. It is considered the gold standard olfactory test and was selected as a result of multiple studies having demonstrated its sensitivity to smoking habits, gender, race, age and a range of olfactory disorders [26, 41, 42], along with its test-retest reliability coefficients in excess of $r=0.9$ [41]. It also has the ability to be self-administered, facilitating cost-effectiveness, practicality and convenience.

Secondly, Sniffin' Sticks test was selected. There are two variations of this test. Sniffin' Sticks 16 comprises 16 reusable odour-dispensing pens that are subdivided to assess odour threshold (T), discrimination (D) and identification (I), later amalgamated into a composite TDI score. The test has been published in well over 200 articles and has a test-retest reliability of $r=0.92$ (T), $r=0.80$ (D), $r=0.88$ (I) [43]. Owing to being a shorter test, it has the advantage of reducing potential olfactory fatigue and loss of attention which may be a factor in $\mathrm{MCI}$ and $\mathrm{AD}$. Furthermore, subcategories provide the advantage of comprehensively evaluating each olfactory domain, in comparison to the UPSIT which only tests olfactory identification. Sniffin' Sticks 12 comprises 12 odour-dispensing pens and, like the UPSIT, focuses exclusively on odour identification.

\section{Critical Appraisal of Literature}

As both cross-sectional studies (CSS) and longitudinal studies (LS) were included in this systematic review, two separate critical appraisal tools were used. Firstly, the Appraisal tool for Cross-Sectional Studies (AXIS) tool was used to appraise individual CSS (see Appendix 1 in the electronic supplementary material). It was developed in 2016 by a select panel 
encompassing a number of different disciplines such as epidemiology and public health. The eventual inclusion criteria were formulated using The Delphi Process, in which each question was agreed upon by at least $80 \%$ of the group. The final product is a critical appraisal tool that accurately assesses reporting quality and bias in CSS [44]. Three questions were removed from the AXIS tool as they were not relevant to the selected studies: 'Were measures undertaken to address and categorise non-responders? Does the response rate raise concerns about non-response bias? If appropriate, was information about non-responders described?'

To critically appraise each LS, the Critical Appraisal Skills Programme (CASP) checklist for cohort studies was used (see Appendix 2 in the electronic supplementary material). It comprised a 15-question checklist and was used to assess the quality and bias in the LS selected for the review. Its aim is to critically appraise the validity, results and assess how the results of the study will help locally. Where a study had both an LS and CSS component, the CASP tool was used for critical appraisal. One question was removed because of lack of relevance: 'Was the exposure accurately measured to minimise bias?'

\section{Ethical Compliance}

This article is based on previously conducted studies and does not contain any studies with human participants or animals performed by any of the authors.

\section{RESULTS}

A systematic literature search was performed on 17 October 2019 using the search terms and Boolean operators 'Dementia OR Alzheimer's AND olfaction AND cognitive impairment' yielding 111 articles. Articles were assessed using the inclusion/exclusion criteria as shown by the flowchart in Fig. 1, alongside the Patient, Intervention, Comparison, Outcome (PICO) strategy shown in Table 1.

\section{Study Selection and Eligibility Criteria}

Articles were excluded on the basis of age (greater than 5 years old) in order to review the latest literature $(n=37)$, review articles $(n=13)$, if the predominant neurodegenerative disease being investigated was PD $(n=7)$ or (amyotrophic lateral sclerosis (ALS) $(n=1)$, if participants had co-morbidity $(n=1)$ if not performed on humans $(n=1)$, or were unable to be readily attained $(n=4)$. Therefore 47 full-text articles were assessed for eligibility, and further excluded based on ineligible study design or no comparable group $(n=25)$ or the UPSIT or Sniffin' Sticks olfactory assessment tool not being used $(n=13)$, leaving 9 studies with a total of 14,760 participants for inclusion in this systematic review (Table 2).

\section{Conclusions of Selected Articles}

Despite different study designs, age, follow-up, olfactory and cognitive measures used, olfactory function was strongly correlated with cognitive function (Table 3). These results are in alignment with previously published literature on this topic. LS allowed change to be witnessed over time and provide more accurate insight into how MCI progressed into AD. However, Yahiaoui-Doktor et al. state that odour identification alone was not sufficient to discriminate between those with or without cognitive impairment, which is the only included study unable to do this [54].

\section{Bias}

\section{Selection Bias}

Of the included nine articles, five recruited participants with MCI and AD from specialist services such as a memory clinic $[47,49,51-53]$ and healthy controls (HC) from the general community or were unstated. Devanand et al. only recruited Medicare beneficiaries [46]. As demonstrated in Table 4, Velayudhan et al. included nine domestic partners and four firstdegree relatives of the participants with $\mathrm{MCI} /$ $\mathrm{AD}$ in the HC cohort [53]. 

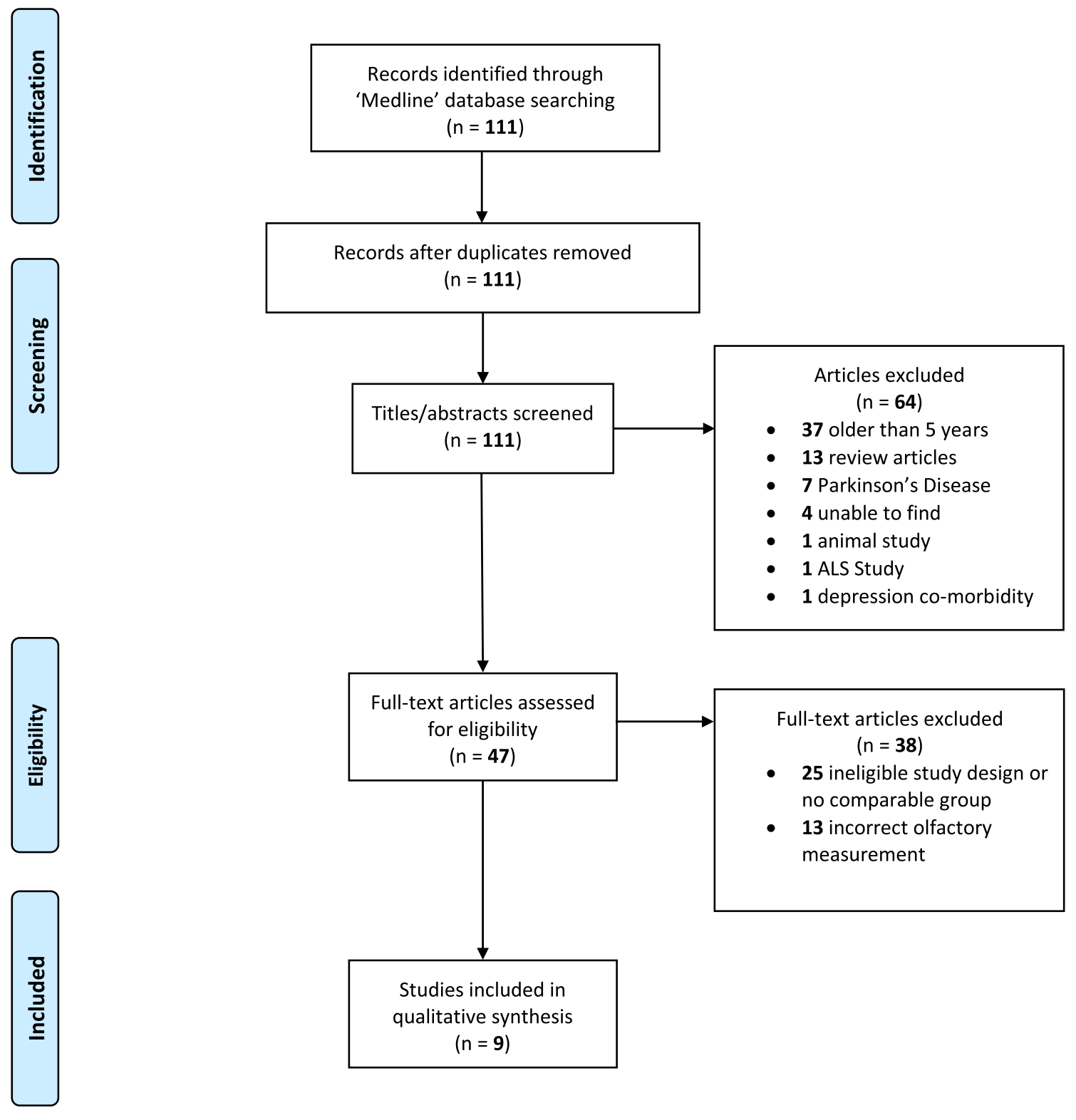

Fig. 1 PRISMA flowchart of selected studies included in this systematic review [45]

\section{Sampling Bias}

There was wide variability in the exclusion criteria of selected articles. Table 5 demonstrates that only three of nine studies considered infection in their exclusion criteria $[48,51,53]$, only two considered sinonasal disease $[51,53]$ and two considered head trauma [51, 52]. Smoking is only included in the exclusion criteria in three articles $[49,51,53]$.

\section{Cultural Bias}

As demonstrated in Table 2, four of the nine articles included in this review used Sniffin' Sticks in a cultural environment in which it was not validated [47, 50-52]. Furthermore, the UPSIT was used on UK participants in a study by Velayudhan et al. despite lack of validity [53]. 
Table 1 PICO framework used to guide research question

\begin{tabular}{lll}
\hline Acronym & Definition & Determinants \\
\hline P & $\begin{array}{c}\text { Patient or } \\
\text { population }\end{array}$ & Patient's with AD \\
I & Intervention & $\begin{array}{c}\text { Preclinical detection of olfactory disturbance by the following olfactory assessment tools: } \\
\text { University of Pennsylvania Smell Identification Test (UPSIT) and Sniffin' Sticks }\end{array}$ \\
C & $\begin{array}{c}\text { Control or } \\
\text { comparison }\end{array}$ & Adult participants without AD or mild cognitive impairment \\
& Outcome & How preclinical olfactory changes correlate with diagnosis/progression of cognitive decline \\
\hline
\end{tabular}

PICO framework demonstrating the focus of this systematic review. AD was selected as a result of being the most prevalent neurodegenerative disease and therefore progress in this area would benefit the widest population of patients. UPSIT and Sniffin' Sticks were selected because they are the most commonly used olfactory tests in the literature and provide the widest amount of data for critical appraisal

\section{Covariates}

Age, sex and education were included as a covariate in each of the studies. As shown by Table 6, apolipoprotein E (APOE) $\varepsilon 4$ was considered in three of the nine studies [48-50]. Kreisl et al. found no correlation between UPSIT scores and APOE $\varepsilon 4$ status (OR 1.75; 95\% CI $0.56,5.44 ; p=0.3352$ ), and also that APOE $\varepsilon 4$ status was not significantly higher in MCI in comparison to HC ( $p=0.4488)$ [49]. Palta et al. also found that APOE $\varepsilon 4$ status did not modify the association between OI and MCI [50]. Woodward et al. found that APOE $\varepsilon 4$ status was higher in both MCI and AD when compared to HC; however, odour identification deficiency was independent from APOE $\varepsilon 4$ status [48]. Furthermore, smoking is included as a covariate in four articles [46, 48, 50, 51]

\section{Medication Use of Participants}

Table 7 shows that only three of the nine included articles considered medication use in their study design or as a covariate $[49,50,52]$, and one stated lack of consideration as a limitation [48]. Even when medication use was included in the study design, there was typically only consideration of a specific type, such as psychotropics, and did not account for all medications a participant may be taking. In all other studies there was no mention of the positive or negative correlation that medication use can have on olfactory function.

\section{DISCUSSION}

\section{Conclusions Summary}

Despite different study designs, follow-up times, use of olfactory tests and cognitive outcome measures, all studies included in this review found a correlation between OI and cognitive decline. These findings support the use of olfactory testing as a supplementary tool for use alongside current testing criteria to further refine diagnostic accuracy. As a result of OI being prevalent before cognitive decline becomes evident, results show that it could be a useful tool for accurately assessing patients who may be at increased risk of later progression to AD. This suggests the potential for modifiable therapies to be developed before symptoms become prevalent. Although Yahiaoui-Doktor et al. were unable to discriminate cognitive impairment using odour identification alone, they state that longitudinal analysis would show which olfactory testing predicts future risk of cognitive impairment [54]. 


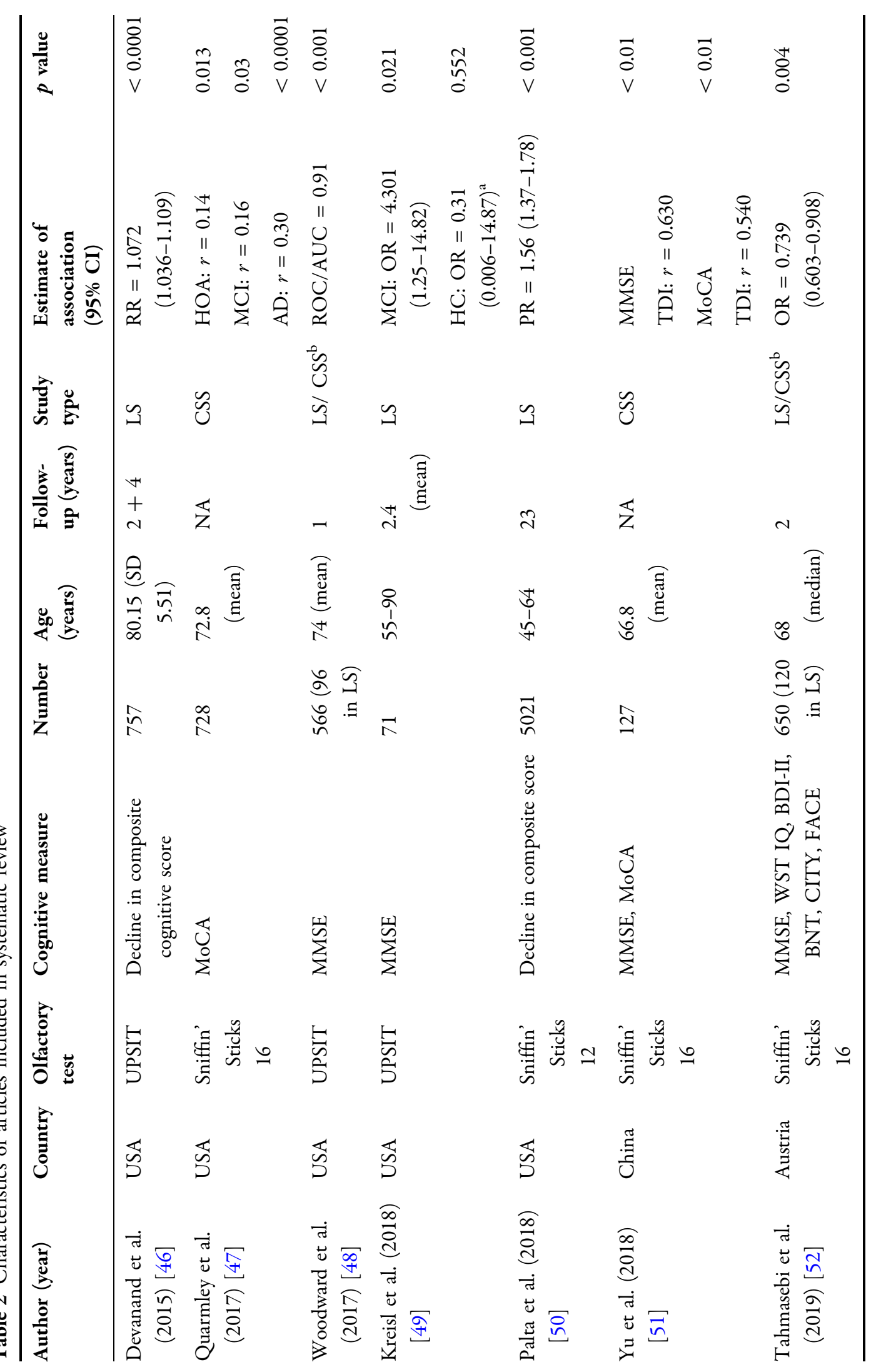




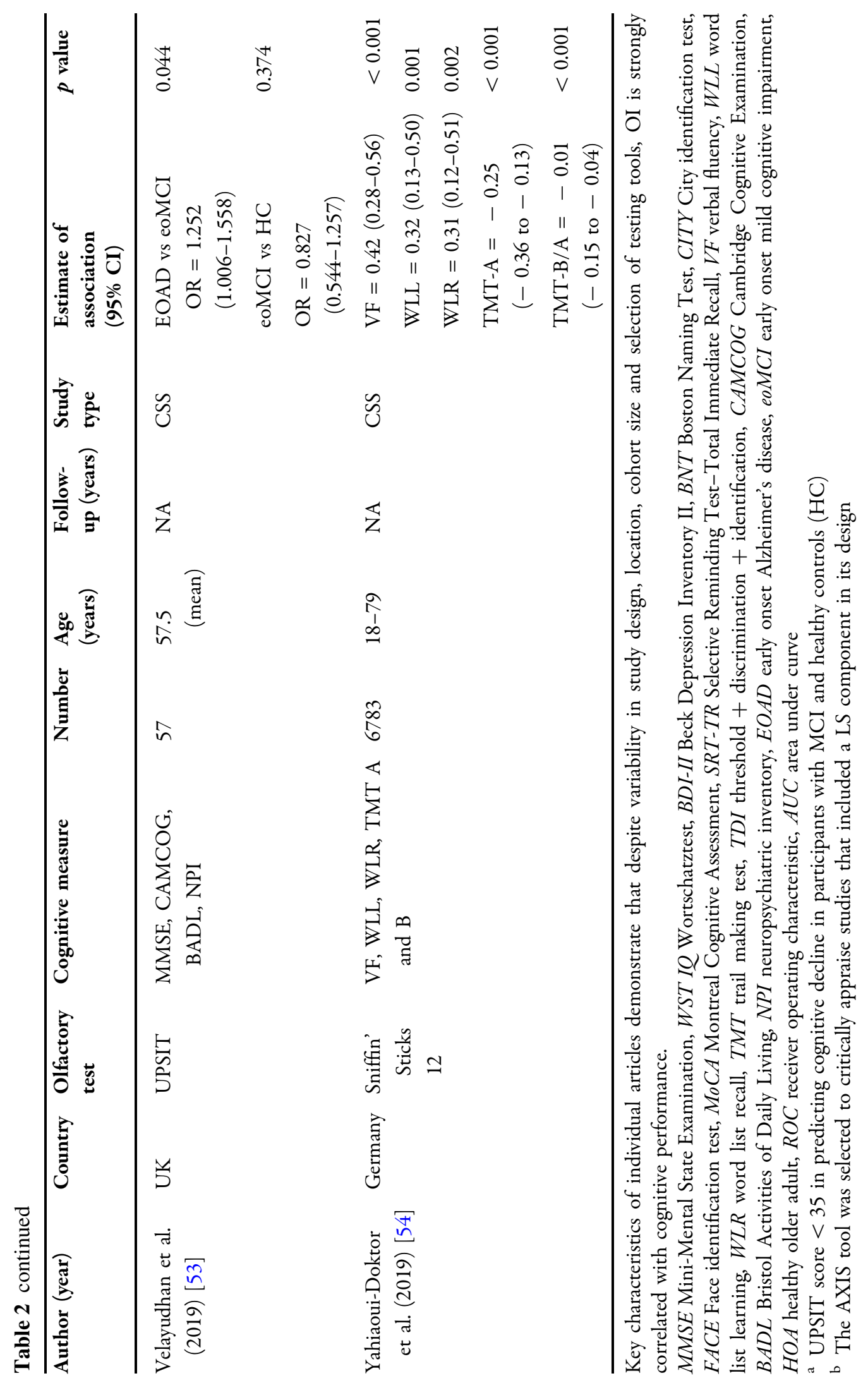


Table 3 Conclusions summary of included articles

\begin{tabular}{|c|c|}
\hline Authors & Conclusions \\
\hline Devanand et al. [46] & $\begin{array}{l}\text { Olfactory identification testing was superior than verbal episodic memory testing in predicting } \\
\text { future cognitive decline in cognitively intact participants }\end{array}$ \\
\hline Quarmley et al. [47] & Olfactory testing is a useful supplementary screening tool in clinical categorisation of $\mathrm{AD}$ and $\mathrm{MC}$ \\
\hline Woodward et al. [48] & $\begin{array}{l}\text { Olfactory identification provides a useful screening tool for AD-related amnestic disorder, and can } \\
\text { be used to stratify risk of conversion from aMCI to AD }\end{array}$ \\
\hline Kreisl et al. [49] & $\begin{array}{l}\text { Participants with high UPSIT predicted absence of amyloidosis on PET imaging with } 100 \% \\
\text { negative predictive value, but only } 41 \% \text { positive predictive value. This shows that UPSIT has the } \\
\text { potential to determine who should undergo PET scanning to further refine diagnostic accuracy. } \\
\text { Participants with high UPSIT scores are less likely to experience memory decline }\end{array}$ \\
\hline Palta et al. $[50]$ & $\begin{array}{l}\text { Reduced olfactory function was associated with lower cognitive performance across multiple } \\
\text { domains, showing a higher overall prevalence of MCI }\end{array}$ \\
\hline Yu et al. [51] & $\begin{array}{l}\text { Olfactory dysfunction is seen in patients with } \mathrm{AD} \text {, demonstrated by overall declines in all } 3 \\
\text { olfactory domains }\end{array}$ \\
\hline Tahmasebi et al. [52] & $\begin{array}{l}\text { Objective olfactory assessments are a promising aid in helping to predict conversion from MCI to } \\
\text { AD. However, low sensitivity and high specificity mean a combining olfactory testing with } \\
\text { neuropsychological testing will be far more beneficial }\end{array}$ \\
\hline Velayudhan et al. [53] & $\begin{array}{l}\text { OI seen in participants with EOAD when compared with eoMCI and HC. OI significantly } \\
\text { correlated with diagnosis of EOAD }\end{array}$ \\
\hline $\begin{array}{l}\text { Yahiaoui-Doktor et al. } \\
\qquad \text { [54] }\end{array}$ & $\begin{array}{l}\text { Significant correlation between olfactory and cognitive performance. However, olfactory } \\
\text { identification alone was not sufficient to discriminate between participants with or without } \\
\text { cognitive impairment }\end{array}$ \\
\hline
\end{tabular}

Brief conclusion summary of individual articles included in this systematic review. All articles found a correlation between $\mathrm{OI}$ and $\mathrm{MCI}$ and/or $\mathrm{AD}$, suggesting its value as a supplementary tool alongside other diagnostic testing

EOAD early onset Alzheimer's disease, eoMCI early onset mild cognitive impairment, aMCI amnestic mild cognitive impairment, $H C$ healthy control, $O I$ olfactory impairment, $M C I$ mild cognitive impairment

\section{Bias}

\section{Selection Bias}

Sampling bias was seen in multiple studies. Five recruited participants with $\mathrm{MCI}$ and $\mathrm{AD}$ from specialist services such as a memory clinic, hospital or medical professional referral $[47,49,51-53]$. Devanand et al. only recruited Medicare beneficiaries, which may not accurately represent the entire population [46]. HC were recruited from the general community or unstated, representing an inherent selection bias. The most significant bias was seen in the study by Velayudhan et al. Of 21 HC participants, 9 were domestic partners of the participants with MCI/AD, despite evidence showing a significantly increased risk of dementia when living with a spouse with the disease [55]. Furthermore, four first-degree relatives of the participants with MCI/AD were also included in the HC cohort despite research showing the statistically significant genetic correlation of AD [56]. This significantly effects the results when considering the small overall cohort size. Participants were largely screened from a single community; however, YahiaouiDoktor et al. included participants from an entire German city [54] and Palta et al. included 
Table 4 Recruitment of participants

\begin{tabular}{|c|c|c|c|}
\hline Authors & HC & MCI & AD \\
\hline Devanand et al. [46] & \multicolumn{3}{|c|}{ All participants Medicare beneficiaries recruited from North Manhattan-WHICAP } \\
\hline Quarmley et al. [47] & Unstated & \multicolumn{2}{|l|}{ Memory clinic } \\
\hline Woodward et al. [48] & Unstated & & \\
\hline Kreisl et al. [49] & Public advertisement & Memory clinic & - \\
\hline Palta et al. [50] & \multicolumn{3}{|c|}{4 US communities (Maryland, North Carolina, Minnesota, Mississippi) } \\
\hline Yu et al. [51] & Recruited from the local community & $\begin{array}{l}\text { Departments of } \\
\text { Tiantan Hosp }\end{array}$ & Neurology, Beijing \\
\hline Tahmasebi et al. [52] & Public advertisement & Referred by me & al or self-referred \\
\hline Velayudhan et al. [53] & $\begin{array}{l}\text { Recruited from a group of healthy } \\
\text { volunteers ( } 9 \text { domestic partners, } 4 \\
\text { first-degree relatives, and } 8 \text { unrelated } \\
\text { volunteers) }\end{array}$ & YODAS, MHS & \\
\hline Yahiaoui-Doktor et al. [54] & \multicolumn{3}{|c|}{ Randomly selected adults from Leipzig, Germany } \\
\hline
\end{tabular}

Demonstrating how each subcategory of participants was recruited for inclusion into each individual study. There is wide variability in the recruitment process, introducing selection bias into multiple studies

$H C$ healthy control, $M C I$ mild cognitive impairment, $A D$ Alzheimer's disease, YODAS Young Onset Dementia Assessment Service, MHSOP mental health services for older people, WHICAP Washington Heights/Inwood Columbia Aging Project

participants from four separate US states [50], therefore largely negating selection bias.

\section{Sampling Bias}

A study of patients aged $16-75$ by Chen et al. concluded that URTI, sinonasal diseases (NSD) and trauma account for $70 \%$ of OI [57]. Despite this, Table 5 demonstrates that only three of nine studies considered infection in their exclusion criteria [48, 51, 53], only two considered sinonasal disease $[51,53]$ and two considered head trauma [51, 52]. Smoking is also known to cause a decrease in olfaction; however, a systematic review by Ajmani et al. concluded that ex-smokers were found to have the same function as those that never smoked, suggesting a reversibility on cessation [58]. Again, current or previous smokers were only included in the exclusion criteria in the minority of articles $[49,51,53]$ and considered as covariates in four others [46, 48, 50, 51]. As the evidence demonstrates the many factors that influence olfactory function, it seems that having a minimum standard of inclusion/ exclusion criteria across all future studies would facilitate increasingly accurate conclusions of the associations between $\mathrm{OI}$ caused by $\mathrm{AD}$ pathology alone.

\section{Cultural Bias}

Of the nine articles included in this review, five use an olfactory test in a cultural environment in which it was not validated. Sniffin' Sticks was used by Tahmasebi et al. on Austrian participants [52], Quarmley [47] and Palta et al. [50] on American participants, and $\mathrm{Yu}$ et al. [51] on Chinese participants. However, Sniffin' Sticks was developed in Germany with the intention of providing an accurate assessment for the European population. The evidence shows that this has not been achieved, with a recent study from Denmark concluding that it was not validated on a Danish population [59]. If Sniffin' Sticks cannot be validated for use in a neighbouring country, then how applicable is its use on participants on a different continent? It could be argued that despite European countries sharing many factors, each has a unique culture 
Table 5 Exclusion criteria of selected articles

Authors Exclusion criteria

Devanand et al. [46] Stroke

PD

Quarmley et al. [47] Unstated

Woodward et al. [48] Nonamnestic MCI $(n=10)$

Hachinski score $>4$ and clinical or imaging evidence of a stroke

Active cold or allergies

Control participants underwent neuropsychological testing (NPT) and were excluded if any measure had a $Z$ score $<-1.5$

Kreisl et al. [49] Stroke or radiographic evidence of cortical or large subcortical infarct

Impairment due to medical conditions or medications

Specific neurological diagnoses (e.g., PD, epilepsy)

Alcohol or drug abuse or dependence

Current major depressive disorder or history of psychosis

Smoking history

UPSIT score $<14$ (to avoid confounding effect from congenital anosmia)

Palta et al. [50] Non-black/non-white participants $(n=18)$

Black participants from Minneapolis, Minnesota, and Washington County, Maryland ( $n=25)$

Participants who were missing the smell test data $(n=440)$

Participants with diagnosed dementia $(n=247)$ were excluded from the analysis

Yu et al. [51] Acute respiratory infections within 3 weeks

Chronic nasitis and sinusitis, and chronic obstructive pulmonary disease (COPD)

Long-term or significant exposure to volatile substances, such as pesticides, herbicides, metallic dusts, acid fumes, industrial solvents, cleaning products or sawdust

Severe head trauma, nasal surgery

Smoking and drug abuse

Other neuropsychiatric disorders affecting olfactory function, such as PD, multiple sclerosis (MS) and epilepsy

Tahmasebi et al. [52] Stroke

History of severe head injury

Current psychiatric diagnosis according to ICD-10

Any medical condition that leads to severe cognitive deterioration including renal, respiratory, cardiac and hepatic disease 
Table 5 continued

\begin{tabular}{|c|c|}
\hline Authors & Exclusion criteria \\
\hline \multirow[t]{6}{*}{ Velayudhan et al. [53] } & Dementia other than $\mathrm{AD}$ \\
\hline & History of psychiatric disorder, including substance abuse \\
\hline & Neurological illness \\
\hline & Significant unstable systematic illness, and organ failure \\
\hline & History at all of cigarette smoking or had stopped smoking for 20 years or more \\
\hline & $\begin{array}{l}\text { Acute or chronic medical conditions that could affect cerebral functioning or other conditions } \\
\text { known to affect olfactory functioning, such as the common cold or polyps }\end{array}$ \\
\hline \multirow{4}{*}{$\begin{array}{l}\text { Yahiaoui-Doktor et al. } \\
\text { [54] }\end{array}$} & Incomplete smell test $(n=14)$ \\
\hline & Not completed all three tests: VF, TMT A and TMT B/A $(n=73)$ \\
\hline & Missing information on education or depression $(n=395)$ \\
\hline & $\mathrm{PD}(n=16)$ \\
\hline
\end{tabular}

Highlighting the wide variability of exclusion criteria resulting in multiple causes of OI not being considered. This introduces sampling bias and decreases the accuracy in correlation between OI caused by $\mathrm{MCI} / \mathrm{AD}$

Table 6 Covariates considered in selected articles

\begin{tabular}{ll}
\hline Authors & Covariates included \\
\hline Devanand et al. [46] & Age, sex, education, short selective recall test, functional impairment, smoking \\
Quarmley et al. [47] & Age, sex, education, race, MoCA \\
Woodward et al. [48] & Age, sex, education, smoking and APOE $\varepsilon 4$ genotype \\
Kreisl et al. [49] & Age, sex, education, MMSE, APOE $\varepsilon 4$ genotype \\
Palta et al. [50] & Age, sex, education, smoking status, diabetes mellitus, hypertension and APOE $\varepsilon 4$ genotype \\
Yu et al. [51] & Age, sex, education, smoking, MMSE, MoCA \\
Tahmasebi et al. [52] & Age, sex, education, MMSE \\
Velayudhan et al. [53] & Age, sex, education, MMSE, total CAMCOG score, executive function scores, and BADL \\
Yahiaoui-Doktor et al. [54] & Age, sex, education, depressive symptoms \\
\hline
\end{tabular}

Age, sex and education was considered in each study. Whilst being correlated with AD, APOE $\varepsilon 4$ was not correlated with olfactory score

$B A D L$ Bristol Activities of Daily Living, MMSE Mini Mental State Exam, CAMCOG Cambridge Cognition Examination, $A P O E$ apolipoprotein $\mathrm{E}$

and therefore olfactory tests require subtle modifications to reflect this. Furthermore, the UPSIT was used on UK participants in a study by Velayudhan et al. [53] despite Muirhead et al. demonstrating its lack of validity [60].
Efforts are being made to rectify the issue. The UPSIT has now been culturally modified into 12 different languages such as the Taiwanese UPSIT-CT and a Brazilian-Portuguese UPSIT-Br2 [61]. The theory being that if specific odorants were interchanged to match respective 
Table 7 Medication use in selected articles

\begin{tabular}{|c|c|}
\hline Authors & Medication criteria \\
\hline Devanand et al. [46] & Not considered in design \\
\hline Quarmley et al. [47] & Not considered in design \\
\hline Woodward et al. [48] & Not considered in design, but stated as a limitation \\
\hline Kreisl et al. [49] & $\begin{array}{l}\text { Excluded participants based on medication use (medications which cause cognitive impairment, } \\
\text { but not stated which medications cause this) }\end{array}$ \\
\hline Palta et al. $[50]$ & Considered diabetic and anti-hypertensives use as covariates in data analysis \\
\hline Yu et al. [51] & Not considered in design \\
\hline Tahmasebi et al. [52] & Excluded controls on the basis of psychotropic medication use \\
\hline Velayudhan et al. [53] & Not considered in design \\
\hline $\begin{array}{l}\text { Yahiaoui-Doktor et al. } \\
\quad[54]\end{array}$ & Not considered in design \\
\hline
\end{tabular}

Despite its effect on olfactory function, medication use was only excluded in 2 of the 9 articles, considered as a covariate in 1 , and stated as a limitation in 1 study. If every study had considered medications in their design, more accurate conclusions between OI driven by MCI/AD may have been demonstrated

cultures, UPSIT scores would have a foundation on which to be compared internationally. Similar work is being carried out using the Sniffin' Sticks test. An adapted version has been created for a Lithuanian population [62], and after the Danish study discovered its lack of validity [59], they modified each odour descriptor until more than $75 \%$ of healthy participants could correctly identify it. This figure is the definition of validation for use on a specific population originally described by Hummel et al. [63]. The only study included in this review that included any variation over the original version was by Devanand et al., allowing Spanish-speaking participants the option of the Spanish version of the UPSIT [46]. Using the standard test over a culturally modified one is perhaps the single biggest error in design seen across the majority of the included studies.

\section{Covariates}

Age and sex are proven to cause a change in olfactory function [64-67], and all studies controlled for these covariates [46-54]. Despite APOE $\varepsilon 4$ being the main genetic risk factor for $\mathrm{AD}$ [68], and previous work by Graves et al. showing its significance [69], it did not necessarily correlate with decreased olfactory function in the studies which included it in the design. One possible reason for this discrepancy is the use of olfactory test chosen for the study. Graves et al. chose the Cross-Cultural Smell Identification Test (CC-SIT), which is subset of the UPSIT comprising 12 odorants. Evidence shows that internal consistency reliability (ICR) decreases when using progressively smaller fractions of the overall 40-item UPSIT [70]. This results in decreased accuracy in detecting OI and therefore less reliable conclusions of association.

\section{Medication Use}

Only four studies included in this review address medication use $[48-50,52]$. Table 7 demonstrates that Kreisl et al. excluded participants on the basis of cognitive impairment induced by medication use; however, they do not state what medications may cause this [49]. Secondly, Palta et al. considered diabetic and anti-hypertensives use as covariates in their analysis of the data [50]. Finally, Tahmasebi et al. excluded controls on the basis of 
psychotropic medication use [52]. Woodward et al. did not include medication use in study design; however, they acknowledged that 'prescription drug use can affect olfactory function' as a limitation [48].

This is a weakness across the studies as medications are proven to affect olfactory function. Schiffman highlighted that $50 \%$ of the top 100 medications used in the USA have the potential to elicit chemosensory side effects [71]. This is echoed by Lötsch et al. who report 71 medications that both positively and negatively modulate olfactory function [72]. An example being levothyroxine, which is positively correlated with an increase in test score, while the opioid remifentanil caused OI in healthy volunteers [73]. Often these results are predictable. All types of opioid receptors are found in the olfactory system, with the highest density of delta receptors in the central nervous system being found in the olfactory bulb [74]; therefore, a logical connection can be made between opioid use and olfactory function. However, as other medications elicit their effects via different pathways, it becomes increasingly difficult to predict their augmentation of olfactory function. Currently, the effects of medications on olfaction are not part of the requirements of drug development, and when considering that $22.8 \%$ of UK residents aged over 69 years take more than five medications [75], it seems an opportunity to understand impairment is being ignored. As the majority of participants in this systematic review were older than 60 years of age, the evidence suggests that a significant proportion are taking regular medications. Despite the difficulty in predicting how this would affect olfactory function, there needs to be increased awareness and consideration into how this is incorporated into study design to further isolate AD pathology as the driver of OI.

\section{What is the Benefit to the Individual?}

Thus far the discussion has focused on the evolution of an olfactory test that provides an accurate diagnostic marker of preclinical AD. It implicitly assumes that this will be a positive discovery, a step forward in the treatment of such a prevalent and ever-increasing pathology. Preclinical detection empowers the individual to plan for the future, providing the information required to put affairs in order, create an Advance Care Plan, reduce the social cost for assistance and help researchers understand more about AD. Furthermore, early diagnosis allows the individual to become involved with interventional research such as non-invasive brain stimulation, as well as understanding the behavioural and psychological symptoms (BPSD) of AD and how these might be managed in the future. Early detection also facilitates the opportunity to develop treatments that may slow or halt disease progression altogether, providing significant benefit to the individual and society at large. Finally, Howe states that providing the option of testing respects patient autonomy and enhances patient care [76].

\section{Limitations, Strengths and Recommendations}

There were several limitations to this review. Firstly, only one database was searched and a small number of articles were included. By searching multiple databases, it is possible that further studies would have been identified for inclusion. Secondly, there was wide variation in the sample size from 57 to 6783. Including studies with a higher number of participants would facilitate more accurate conclusions. Thirdly, only one study used pathophysiological biomarkers. Fourthly, there was high variability in which covariates were considered in each study design. Study designs considering important covariates would allow OI caused purely by early neurodegenerative pathology to be more readily ascertained. Only five of the nine studies were LS, which limited the evidence of change over time [46, 48-50, 52]. Furthermore, six of the nine studies used olfactory tests focused entirely on identification', and excluding the other two domains may have resulted in loss of important data on how other domains are affected $[46,48-50,53,54]$. 
By including different study designs, sample sizes, a variety of countries and different olfactory tests, there was an opportunity to analyse the correlation between olfactory impairment and cognitive ability across a variety of different study designs. This allowed the association to become self-evident under a variety of conditions. Conclusions based on the synthesis of the data should be tempered by recognition of the limitations identified.

This review highlights the inconsistency in screening, as well as factors considered in statistical analysis and recommends that future research consider these factors to come to increasingly accurate conclusions which may facilitate olfactory testing becoming a part of regular clinical use.

Another key factor to consider in future work is the metabolic differences between early onset $\mathrm{AD}(\mathrm{EOAD})$ and late-onset $\mathrm{AD}$ (LOAD). Chiaravalloti et al. demonstrate that in EOAD, glucose consumption is significantly increased in the left parietal lobe when compared to LOAD [77]. Therefore, to establish the usefulness of olfactory testing as a biomarker of future pathology, future work needs to deepen all possible differences that can occur in such a heterogeneous disease. This may be achieved by stratifying factors such as disease duration and age of onset, which will provide an increasingly accurate assessment of the reliability of olfactory testing.

\section{CONCLUSION}

The results of this systematic review highlight a consistency across different studies in the role of OI in predicting cognitive decline. Each article concluded that olfactory testing provided valuable supplementary data on which diagnosis can be more accurately provided. However, conclusions are being made without fully examining factors that impair olfactory function outside of neurodegenerative disease. There is a need to establish a minimum set of inclusion/exclusion criteria to consistently design studies considering these factors. This may guide future research to refine the accuracy of neuronal pathology being the driver of OI.

\section{ACKNOWLEDGEMENTS}

Funding. No sponsorship was received for this study. A fee waiver was given by Neurology and Therapy for its Rapid Service Fee.

Authorship. All named authors meet the International Committee of Medical Journal Editors (ICMJE) criteria for authorship for this article, take responsibility for the integrity of the work as a whole, and have given their approval for this version to be published.

Medical Writing, Editorial, and other Assistance. Proofreading in the preparation of this article was provided by Rebekah Rajiah.

Disclosures. Rikki L. Winchester and Dr Kathy Martyn have nothing to disclose.

Compliance with Ethics Guidelines. This article is based on previously conducted studies and does not contain any studies with human participants or animals performed by any of the authors.

Data Availability. All data generated or analysed during this study are included in this published article/as supplementary information files.

Open Access. This article is licensed under a Creative Commons Attribution-NonCommercial 4.0 International License, which permits any non-commercial use, sharing, adaptation, distribution and reproduction in any medium or format, as long as you give appropriate credit to the original author(s) and the source, provide a link to the Creative Commons licence, and indicate if changes were made. The images or other third party material in this article are included in the article's Creative Commons licence, unless indicated otherwise in a credit line to the material. If material is not included in the article's Creative Commons licence and your intended use is not permitted by statutory regulation or exceeds the permitted use, you will need to obtain permission directly from the copyright holder. To view a copy of this licence, 
visit http://creativecommons.org/licenses/bync/4.0/.

\section{REFERENCES}

1. Zou Y, Lu D, Liu L, Zhang H, Zhou Y. Olfactory dysfunction in Alzheimer's disease. Neuropsychiatr Dis Treat. 2016;12:869-75. https://doi.org/10.2147/ NDT.S104886.

2. Alzheimer's Society. Facts for the media. https:// www.alzheimers.org.uk/about-us/news-and-media/ facts-media. Accessed 31 Oct 2019.

3. National life tables, UK-Office for National Statistics. https://www.ons.gov.uk/peoplepopulationand community/birthsdeathsandmarriages/lifeexpectan cies/bulletins/nationallifetablesunitedkingdom/ 2015to2017. Accessed 31 Oct 2019.

4. McKhann G, Drachman D, Folstein M, Katzman R, Price D, Stadlan EM. Clinical diagnosis of Alzheimer's disease. Neurology. 1984;34:939. https://doi. org/10.1212/WNL.34.7.939.

5. Knopman DS, DeKosky ST, Cummings JL, et al. Practice parameter: diagnosis of dementia (an evidence-based review). Report of the Quality Standards Subcommittee of the American Academy of Neurology. Neurology. 2001;56(9):1143-53. https://doi.org/10.1212/WNL.56.9.1143.

6. Dubois B, Feldman HH, Jacova C, et al. Research criteria for the diagnosis of Alzheimer's disease: revising the NINCDS-ADRDA criteria. Lancet Neurol. 2007;6(8):734-46. https://doi.org/10.1016/ S1474-4422(07)70178-3.

7. Dubois B, Feldman HH, Jacova C, et al. Revising the definition of Alzheimer's disease: a new lexicon. Lancet Neurol. 2010;9(11):1118-27. https://doi. org/10.1016/S1474-4422(10)70223-4.

8. McKhann GM, Knopman DS, Chertkow H, et al. The diagnosis of dementia due to Alzheimer's disease: recommendations from the National Institute on Aging-Alzheimer's Association workgroups on diagnostic guidelines for Alzheimer's disease. Alzheimers Dement. 2011;7(3):263-9. https://doi.org/ 10.1016/j.jalz.2011.03.005.

9. Sperling RA, Aisen PS, Beckett LA, et al. Toward defining the preclinical stages of Alzheimer's disease: recommendations from the National Institute on Aging-Alzheimer's Association workgroups on diagnostic guidelines for Alzheimer's disease. Alzheimers Dement. 2011;7(3):280-92. https://doi. org/10.1016/j.jalz.2011.03.003.
10. Jack CR, Bennett DA, Blennow K, et al. NIA-AA research framework: toward a biological definition of Alzheimer's disease. Alzheimers Dement. 2018;14(4):535-62. https://doi.org/10.1016/j.jalz. 2018.02.018.

11. Schneider JA, Aggarwal NT, Barnes L, Boyle P, Bennett DA. The neuropathology of older persons with and without dementia from community versus clinic cohorts. J Alzheimers Dis. 2009;18(3): 691-701. https://doi.org/10.3233/JAD-2009-1227.

12. NICE. Dementia diagnosis and assessment. March 2018. https://pathways.nice.org.uk/pathways/ dementia/dementia-diagnosis-and-assessment\# content=view-node:nodes-diagnosis-and-assessment. Accessed 5 Oct 2018.

13. Alladi S, Xuereb J, Bak T, et al. Focal cortical presentations of Alzheimer's disease. Brain. 2007;130(10):2636-45. https://doi.org/10.1093/ brain/awm 213 .

14. NICE. Dementia: assessment, management and support for people living with dementia and their carers. https://www.nice.org.uk/guidance/ng97/ chapter/Recommendations. Accessed 23 Jan 2020.

15. Windon MJ, Kim SJ, Oh ES, Lin SY. Predictive value of olfactory impairment for cognitive decline among cognitively normal adults. Laryngoscope. 2019. https://doi.org/10.1002/lary.28166.

16. Devanand DP. Olfactory identification deficits, cognitive decline, and dementia in older adults. Am J Geriatr Psychiatry. 2016;24(12):1151-7. https:// doi.org/10.1016/j.jagp.2016.08.010.

17. Waldton S. Clinical observations of impaired cranial nerve function in senile dementia. Acta Psychiatr Scand. 1974;50(5):539-47. https://doi.org/ 10.1111/j.1600-0447.1974.tb09714.x.

18. Murphy C, Schubert CR, Cruickshanks KJ, Klein BEK, Klein R, Nondahl DM. Prevalence of olfactory impairment in older adults. JAMA. 2002;288(18): 2307-12. https://doi.org/10.1001/jama.288.18. 2307.

19. Doty RL, Shaman P, Applebaum SL, Giberson R, Siksorski L, Rosenberg L. Smell identification ability: changes with age. JSTOR. 1984;226(4681): 1441-1443. https://www-jstor-org.ezproxy.sussex. ac.uk/stable/1693918. Accessed 31 Oct 2019.

20. Pence TS, Reiter ER, DiNardo LJ, Costanzo RM. Risk factors for hazardous events in olfactory-impaired patients. JAMA Otolaryngol Neck Surg. 2014; 140(10):951-5. https://doi.org/10.1001/jamaoto. 2014.1675. 
21. Stevenson RJ. An initial evaluation of the functions of human olfaction. Chem Senses. 2010;35(1):3-20. https://doi.org/10.1093/chemse/bjp083.

22. Pinto JM, Wroblewski KE, Kern DW, Schumm LP, McClintock MK. Olfactory dysfunction predicts 5-year mortality in older adults. PLoS One. 2014. https://doi.org/10.1371/journal.pone.0107541.

23. Gopinath B, Sue CM, Kifley A, Mitchell P. The association between olfactory impairment and total mortality in older adults. J Gerontol A Biol Sci Med Sci. 2012;67A(2):204-9. https://doi.org/10.1093/ gerona/glr165.

24. Sullivan RM, Wilson DA, Ravel N, Mouly A-M. Olfactory memory networks: from emotional learning to social behaviors. Front Behav Neurosci. 2015. https://doi.org/10.3389/fnbeh.2015.00036.

25. Miwa T, Furukawa M, Tsukatani T, Costanzo RM, DiNardo LJ, Reiter ER. Impact of olfactory impairment on quality of life and disability. Arch Otolaryngol Neck Surg. 2001;127(5):497-503. https:// doi.org/10.1001/archotol.127.5.497.

26. Doty RL, Reyes PF, Gregor T. Presence of both odor identification and detection deficits in Alzheimer's disease. Brain Res Bull. 1987;18(5):597-600. https:// doi.org/10.1016/0361-9230(87)90129-8.

27. Australian Medical Association. Guide to the assessment of the degree of permanent impairment. 2011;2(1):83. https://www.comcare.gov.au/about/ forms-publications/documents/publications/claims /assessment-of-degree-of-permanent-impairment. pdf.

28. Growdon ME, Schultz AP, Dagley AS, et al. Odor identification and Alzheimer disease biomarkers in clinically normal elderly. Neurology. 2015;84(21): 2153-60. https://doi.org/10.1212/WNL.000000 0000001614.

29. Lafaille-Magnan M-E, Poirier J, Etienne P, et al. Odor identification as a biomarker of preclinical AD in older adults at risk. Neurology. 2017;89(4): 327-35. https://doi.org/10.1212/WNL.000000000 0004159 .

30. Doty RL. Olfaction in Parkinson's disease and related disorders. Neurobiol Dis. 2012;46(3):527-52. https://doi.org/10.1016/j.nbd.2011.10.026.

31. Bowman GL. Biomarkers for early detection of Parkinson disease: a scent of consistency with olfactory dysfunction. Neurology. 2017;89(14): 1432-4. https://doi.org/10.1212/WNL.000000 0000004383.

32. Fullard ME, Morley JF, Duda JE. Olfactory dysfunction as an early biomarker in Parkinson's disease.
Neurosci Bull. 2017;33(5):515-25. https://doi.org/ 10.1007/s12264-017-0170-X.

33. McShane R, Nagy Z, Esiri M, et al. Anosmia in dementia is associated with Lewy bodies rather than Alzheimer's pathology. J Neurol Neurosurg Psychiatry. 2001;70(6):739-43. https://doi.org/10. 1136/jnnp.70.6.739.

34. McKeith I, Taylor J-P, Thomas A, Donaghy P, Kane J. Revisiting DLB diagnosis: a consideration of prodromal DLB and of the diagnostic overlap with Alzheimer disease. J Geriatr Psychiatry Neurol. 2016;29(5):249-53. https://doi.org/10.1177/08919 88716656083.

35. Duff K, McCaffrey RJ, Solomon GS. The pocket smell test. J Neuropsychiatry Clin Neurosci. 2002;14(2):197-201. https://doi.org/10.1176/jnp. 14.2.197.

36. Doty RL. Olfactory dysfunction in Parkinson disease. Nat Rev Neurol. 2012;8(6):329-39. https://doi. org/10.1038/nrneurol.2012.80.

37. da Costa BR, Jüni P. Systematic reviews and metaanalyses of randomized trials: principles and pitfalls. Eur Heart J. 2014;35(47):3336-45. https://doi. org/10.1093/eurheartj/ehu424.

38. Rice DB, Kloda LA, Levis B, Qi B, Kingsland E, Thombs BD. Are MEDLINE searches sufficient for systematic reviews and meta-analyses of the diagnostic accuracy of depression screening tools? A review of meta-analyses. J Psychosom Res. 2016;87: 7-13. https://doi.org/10.1016/j.jpsychores.2016.06. 002 .

39. van Enst WA, Scholten RJPM, Whiting P, Zwinderman AH, Hooft L. Meta-epidemiologic analysis indicates that MEDLINE searches are sufficient for diagnostic test accuracy systematic reviews. J Clin Epidemiol. 2014;67(11):1192-9. https://doi.org/10. 1016/j.jclinepi.2014.05.008.

40. Dunn K, Marshall JG, Wells AL, Backus JEB. Examining the role of MEDLINE as a patient care information resource: an analysis of data from the Value of Libraries study. J Med Libr Assoc. 2017;105(4): 336-46. https://doi.org/10.5195/jmla.2017.87.

41. Doty RL, Shaman P, Dann M. Development of the university of Pennsylvania smell identification test: a standardized microencapsulated test of olfactory function. Physiol Behav. 1984;32(3):489-502. https://doi.org/10.1016/0031-9384(84)90269-5.

42. Doty RL, Shaman P, Kimmelman CP, Dann MS. University of Pennsylvania smell identification test: a rapid quantitative olfactory function test for the clinic. Laryngoscope. 1984;94(2):176-8. https://doi. org/10.1288/00005537-198402000-00004. 
43. Haehner A, Mayer A-M, Landis BN, et al. High testretest reliability of the extended version of the "Sniffin' Sticks" test. Chem Senses. 2009;34(8): 705-11. https://doi.org/10.1093/chemse/bjp057.

44. Downes MJ, Brennan ML, Williams HC, Dean RS. Development of a critical appraisal tool to assess the quality of cross-sectional studies (AXIS). BMJ Open. 2016;6(12):e011458. bmjopen-2016-011458.

45. Moher D, Liberati A, Tetzlaff J, Altman DG. Preferred reporting items for systematic reviews and meta-analyses: the PRISMA statement. BMJ. 2009. https://doi.org/10.1136/bmj.b2535.

46. Devanand DP, Lee S, Manly J, et al. Olfactory deficits predict cognitive decline and Alzheimer dementia in an urban community. Neurology. 2015;84(2):182-9. https://doi.org/10.1212/WNL. 0000000000001132.

47. Quarmley M, Moberg PJ, Mechanic-Hamilton D, et al. Odor identification screening improves diagnostic classification in incipient Alzheimer's disease. J Alzheimers Dis. 2016;55(4):1497-507. https://doi.org/10.3233/JAD-160842.

48. Woodward MR, Amrutkar CV, Shah HC, et al. Validation of olfactory deficit as a biomarker of Alzheimer disease. Neurol Clin Pract. 2017;7(1):5-14. https://doi.org/10.1212/CPJ.0000000000000293.

49. Kreisl WC, Jin P, Lee $S$, et al. Odor identification ability predicts PET amyloid status and memory decline in older adults. J Alzheimers Dis. 2018;62(4):1759-66. https://doi.org/10.3233/JAD170960 .

50. Palta P, Chen H, Deal JA, et al. Olfactory function and neurocognitive outcomes in old age: the atherosclerosis risk in communities neurocognitive study. Alzheimers Dement. 2018;14(8):1015-21. https://doi.org/10.1016/j.jalz.2018.02.019.

51. Yu Q, Guo P, Li D, et al. Olfactory dysfunction and its relationship with clinical symptoms of Alzheimer disease. Aging Dis. 2018;9(6):1084. https://doi. org/10.14336/AD.2018.0819.

52. Tahmasebi R, Zehetmayer S, Pusswald G, Kovacs G, Stögmann E, Lehrner J. Identification of odors, faces, cities and naming of objects in patients with subjective cognitive decline, mild cognitive impairment and Alzheimer's disease: a longitudinal study. Int Psychogeriatr. 2019;31(04):537-49. https://doi.org/10.1017/S1041610218001114.

53. Velayudhan L, Wilson-Morkeh F, Penney E, Jesu AJM, Baillon S, Brugha T. Smell identification function in early-onset Alzheimer's disease and mild cognitive impairment. Int Psychogeriatr.
2019;31(07):1065-70.

https://doi.org/10.1017/ S1041610218001503.

54. Yahiaoui-Doktor M, Luck T, Riedel-Heller SG, Loeffler M, Wirkner K, Engel C. Olfactory function is associated with cognitive performance: results from the population-based LIFE-Adult-Study. Alzheimers Res Ther. 2019;11(1):43. https://doi.org/10. 1186/s13195-019-0494-z.

55. Norton MC, Smith KR, Østbye T, et al. Increased risk of dementia when spouse has dementia? The Cache County Study. J Am Geriatr Soc. 2010;58(5): 895-900. https://doi.org/10.1111/j.1532-5415. 2010.02806.x.

56. Khanahmadi M, Farhud DD, Malmir M. Genetic of Alzheimer's disease: a narrative review article. Iran J Public Health. 2015;44(7):892-901. https://www. ncbi.nlm.nih.gov/pmc/articles/PMC4645760/. Accessed 08 Mar 2020.

57. Chen G, Wei Y, Miao X, Li K, Ren Y, Liu J. Clinical features of olfactory disorders in patients seeking medical consultation. Med Sci Monit. 2013;19: 444-50.

58. Ajmani GS, Suh HH, Wroblewski KE, Pinto JM. Smoking and olfactory dysfunction: a systematic literature review and meta-analysis. Laryngoscope. 2017;127(8):1753-61. https://doi.org/10.1002/lary. 26558.

59. Niklassen AS, Ovesen T, Fernandes H, Fjaeldstad AW. Danish validation of Sniffin' Sticks olfactory test for threshold, discrimination, and identification. Laryngoscope. 2018;128(8):1759-66. https:// doi.org/10.1002/lary.27052.

60. Muirhead N, Benjamin E, Saleh H. Is The University of Pennsylvania Smell Identification Test (UPSIT) valid for the UK population? Otorhinolaryngologist. 2013:99-103. https://theotorhinolaryngologist. co.uk/new/images/pdf/v6_n2/upsit.pdf. Accessed 3 Mar 2020.

61. Silveira-Moriyama L, Azevedo AMS, Ranvaud R, Barbosa ER, Doty RL, Lees AJ. Applying a new version of the Brazilian-Portuguese UPSIT smell test in Brazil. Arq Neuropsiquiatr. 2010;68(5):700-5. https://doi.org/10.1590/S0004-282X201000050000 5.

62. Čičelienė J, Vaičys Ž, Rastenytė D. Development of the Lithuanian version of Sniffin' Sticks 12 odor identification test. Medicina (Mex). 2018. https:// doi.org/10.3390/medicina54020013.

63. Hummel T, Sekinger B, Wolf SR, Pauli E, Kobal G. "Sniffin" Sticks': olfactory performance assessed by the combined testing of odor identification, odor discrimination and olfactory threshold. Chem 
Senses. 1997;22(1):39-52. https://doi.org/10.1093/ chemse/22.1.39.

64. Schubert CR, Cruickshanks KJ, Fischer ME, et al. Olfactory Impairment in an adult population: the Beaver Dam offspring study. Chem Senses. 2012;37(4):325-34. https://doi.org/10.1093/ chemse/bjr102.

65. Doty RL, Kamath V. The influences of age on olfaction: a review. Front Psychol. 2014. https://doi. org/10.3389/fpsyg.2014.00020.

66. Lafreniere D, Mann N. Anosmia: loss of smell in the elderly. Otolaryngol Clin N Am. 2009;42(1):123-31. https://doi.org/10.1016/j.otc.2008.09.001.

67. Chopra A, Baur A, Hummel T. Thresholds and chemosensory event-related potentials to malodors before, during, and after puberty: differences related to sex and age. Neuroimage Amst. 2008;40(3): 1257-63. https://doi.org/10.1016/j.neuroimage. 2008.01.015.

68. Liu C-C, Kanekiyo T, Xu H, Bu G. Apolipoprotein E and Alzheimer disease: risk, mechanisms, and therapy. Nat Rev Neurol. 2013;9(2):106-18. https:// doi.org/10.1038/nrneurol.2012.263.

69. Graves $A B$, Bowen JD, Rajaram L, et al. Impaired olfaction as a marker for cognitive decline: Interaction with apolipoprotein E epsilon4 status. Neurology. 1999;53(7):1480-1480. https://doi.org/10. 1212/WNL.53.7.1480.

70. Doty RL, Frye RE, Agrawal U. Internal consistency reliability of the fractionated and whole University of Pennsylvania Smell Identification Test. Percept Psychophys. 1989;45(5):381-4. https://doi.org/10. 3758/BF03210709.
71. Schiffman SS. Influence of medications on taste and smell. World J Otorhinolaryngol Head Neck Surg. 2018;4(1):84-91. https://doi.org/10.1016/j.wjorl. 2018.02.005.

72. Lötsch J, Knothe C, Lippmann C, Ultsch A, Hummel T, Walter C. Olfactory drug effects approached from human-derived data. Drug Discov Today. 2015;20(11):1398-406. https://doi.org/10.1016/j. drudis.2015.06.012.

73. Lötsch J, Darimont J, Skarke C, Zimmermann M, Hummel T, Geisslinger G. Effects of the opioid remifentanil on olfactory function in healthy volunteers. Life Sci. 2001;69(19):2279-85. https://doi. org/10.1016/S0024-3205(01)01305-4.

74. Schulz S, Schreff M, Koch T, et al. Immunolocalization of two mu-opioid receptor isoforms (MOR1 and MOR1B) in the rat central nervous system. Neuroscience. 1997;82(2):613-22. https://doi.org/ 10.1016/S0306-4522(97)00288-1.

75. Rawle MJ, Richards M, Davis D, Kuh D. The prevalence and determinants of polypharmacy at age 69: a British birth cohort study. BMC Geriatr. 2018. https://doi.org/10.1186/s12877-018-0795-2.

76. Howe EG. Ethical issues in diagnosing and treating Alzheimer disease. Psychiatry Edgmont. 2006;3(5): 43-53. https://www.ncbi.nlm.nih.gov/pmc/ articles/PMC2990623/. Accessed 25 Feb 2020.

77. Chiaravalloti A, Koch G, Toniolo S, et al. Comparison between early-onset and late-onset Alzheimer's disease patients with amnestic presentation: CSF and 18F-FDG PET Study. Dement Geriatr Cogn Disord Extra. 2016;6(1):108-19. https://doi.org/10. $1159 / 000441776$. 